\title{
The vertebrate skeletal muscle thick filaments are not three-stranded. Reinterpretation of some experimental data $^{\star}$
}

\author{
Ludmila Skubiszak $^{\bowtie}$ and Leszek Kowalczyk
}

Laboratory of Computer Simulation of Muscle Contraction, Institute of Biocybernetics and Biomedical Engineering of the Polish Academy of Sciences,

Ks. Trojdena 4, 02-109 Warszawa, Poland

Received: 03 October, 2002; revised: 21 October, 2002; accepted: 13 November, 2002

Key words: myosin molecule packing, thick filament symmetry, mass distribution, Fourier transform, cross-bridge movement

\begin{abstract}
Computer simulation of mass distribution within the model and Fourier transforms of images depicting mass distribution are explored for verification of two alternative modes of the myosin molecule arrangement within the vertebrate skeletal muscle thick filaments. The model well depicting the complete bipolar structure of the thick filament and revealing a true threefold-rotational symmetry is a tube covered by two helices with a pitch of $2 \times 43 \mathrm{~nm}$ due to arrangement of the myosin tails along a helical path and grouping of all myosin heads in the crowns rotated by $240^{\circ}$ and each containing three cross-bridges separated by $0^{\circ}, 120^{\circ}$, and $180^{\circ}$. The cross-bridge crown parameters are verified by EM images as well as by optical and low-angle X-ray diffraction patterns found in the literature. The myosin tail arrangement, at which the C-terminus of about 43-nm length is near-parallel to the filament axis and the rest of the tail is quite strongly twisted around, is verified by the high-angle X-ray diffraction patterns. A consequence of the new packing is a new way of movement of the myosin cross-bridges, namely, not by bending in the hinge domains, but by unwrapping from the thick filament surface towards the thin filaments along a helical path.
\end{abstract}

The new mode for the arrangement of myosin molecules within the vertebrate skeletal muscle thick filaments, introduced by us into the CB (complete bipolar) model (Skubiszak \&
Kowalczyk, 2002), differs considerably from that widely accepted (Squire, 1997; Eakins et al., 2002) and incorporated by us into the TS (three-stranded) model (Skubiszak \&

\footnotetext{
${ }^{\star}$ Preliminary results were presented at the 45th Annual Meeting of the Biophysical Society (Boston, 2001) and the results included in this paper at the 38th Meeting of the Polish Biochemical Society (Wrocław, 2002).

${ }^{\circledR}$ Corresponding author: Ludmila Skubiszak, tel.: (48 22) 6599143 ext. 208; fax: (48 22) 659 7030; e-mail: Ludmila.Skubiszak@ibib.waw.pl
}

Abbreviations: CB, complete bipolar; EM, electron microscopy; LMM, light meromyosin; FFT, logarithmic fast Fourier transform; S2, myosin subfragment-2; TS, three-stranded. 
Kowalczyk, 2002) in two main aspects: (1) the myosin tails are twisted around the filament axis instead to be aligned near parallel; (2) the myosin heads are grouped into asymmetrical instead of symmetrical crowns.

The concept of the straightened myosin tails arranged near-parallel to the filament axis within the filament backbone was originally based on EM observations of various synthetic myosin filaments and filament-like aggregates (Huxley, 1963; Podlubnaya et al., 1969; Harrison et al., 1971; Bennett, 1981; Yagi \& Offer, 1981; Yagi et al., 1981). However, EM allows visualization of the molecule projection in a plane only; any bends or twists cannot be recognized. As a strong argument for the parallel arrangement, specific reflections, meridional at position $1 / 0.51 \mathrm{~nm}^{-1}$ and three equatorial streaks at positions of about $1 / 2.0 \mathrm{~nm}^{-1}, 1 / 1.35 \mathrm{~nm}^{-1}$ and $1 / 1.0 \mathrm{~nm}^{-1}$, observed in high-angle X-ray diffraction patterns, are commonly considered (Cohen \& Holmes, 1963; Elliott et al., 1968; Chew \& Squire, 1995). However, the intensities of the reflections are too weak to conclude that all mass of the myosin tails is distributed parallel to the filament axis.

The question of the cross-bridge configurations, i.e. the mutual rotation of both the three pairs of the myosin heads in a crown and the crowns successively occurring along the filament, is also difficult to resolve unambiguously because no transverse view showing separate cross-bridge crowns exists. The idea of myosin head grouping into symmetrical crowns (Squire, 1972; Squire, 1973; Kensler \& Stewart, 1983) was originally verified on the grounds of the equilateral triangles visible in the transverse view of the H-zones of muscle sarcomeres (Luther \& Squire, 1980). However, in the remaining, much longer part, the thick filament profiles are circular (Craig et al., 1992). Furthermore, a threefold-rotational symmetry of each cross-bridge crown is usually identified with the same symmetry of the whole filament (Kensler \& Stewart, 1983; Kensler \& Woodhead, 1995; Squire, 1997;
Cantino et al., 2002; Eakins et al., 2002). As a strong argument for this, extrapolation of the head array on the surface of relaxed filaments by three symmetrically originated helical tracks with a pitch of $9 \times 14.3 \mathrm{~nm}$ has been recognized. However, it is not possible to precisely extrapolate EM images in such a way; moreover, patterns have been published (Cantino \& Squire, 1986; Cantino et al., 2002) where the head mass distribution clearly reveals a two-stranded feature, never discussed by the authors. Furthermore, resolution of the EM images of isolated thick filaments is usually too low to unambiguously separate the two heads of a myosin molecule; the images support only the notion that the visible subunits are large enough to contain two myosin heads. Because of that the reconstruction methods based on EM images of separate filaments (Stewart \& Kensler, 1986; Eakins et al., 2002) and on low-angle X-ray diffraction patterns of muscles (Hudson et al., 1997) have led to considerably different head positions in relation to the filament backbone. It is also worth noting that the models created by the reconstruction methods are based on the assumption that all the heads are arranged along three symmetrically originated helical paths of a $3 \times 43-\mathrm{nm}$ pitch.

Some thick filament features, inferred on EM grounds, are in direct contrast to those revealed by the optical and low-angle X-ray diffraction methods. This concerns first of all the arrangement of the meridional reflections because they appear not only on the layer lines corresponding to $3 \mathrm{n}$ orders of the $43-\mathrm{nm}$ repeat, i.e. specific for the $9 / 3$ helical symmetry, but also on the 1 st, $2 \mathrm{nd}$, 4 th, 5 th, 7 th, etc. layer lines, i.e. in the so-called "forbidden" positions (Huxley \& Brown, 1967; Haselgrove \& Rodger, 1980; Malinchik \& Lednev, 1992; Bordas et al., 1993). The discrepancies are usually accounted for by different perturbations in the myosin head array on the filament surface. Stewart and Kensler (1986), for instance, suggested that in three successive crowns the heads are localized at different ra- 
dii. Malinchik \& Lednev (1992) concluded that three successive crowns are axially shifted in the range of $43 \mathrm{~nm}$. Levine (1993) interpreted the data by overlapping the heads from different crowns. Haselgrove \& Rodger (1980), Malinchik et al. (1997), as well as Hudson et al. (1997) have attempted to find the proper shape and tilting of each of the two heads in relation to the filament axis. Hudson et al. (1997) used for myosin head the original model devised by Rayment (1993) but to attain a satisfactory consistency of the Fourier transform with low-angle X-ray diffraction patterns the authors shifted the two heads of the myosin molecule so markedly that they became connected to different myosin tails. In all these cases, the symmetrical configuration of each cross- bridge crown was always accepted as being determined by the threefold-rotational symmetry of the whole filament; any other configuration of the cross-bridge crowns has never been considered. Moreover, the descriptions were related either to the myosin tails or to the myosin heads, i.e. the continuity of the myosin molecule has never been explored. As a consequence, no 3D model of a complete bipolar structure of the filament exists, only a fragment of the filament cross-bridge zone, usually $43 \mathrm{~nm}$ long, has been discussed (for a review see: Squire, 1997; Eakins et al., 2002). However, all models based on the assumption of the symmetrical configuration of the cross-bridge crowns lead to an incorrect relation between the number of the cross-bridges protruding from the thick filament backbone and the surrounding thin filaments, namely to the relation nine to six (Skubiszak \& Kowalczyk, 2002) instead of six to six observed experimentally (Yu \& Brenner, 1986; Craig et al., 1992).

Here we present a new approach for verification of two models created on the basis of two alternative modes of the myosin molecule arrangements within the vertebrate skeletal muscle thick filaments, i.e. the complete bipolar model (CB model) and the three-stranded model (TS model) described in the previous paper (Skubiszak \& Kowalczyk, 2002). The verification is based on a comparison of both the calculated mass distribution with that observed in EM images of relaxed thick filaments and the calculated Fourier transform with the diffraction patterns of muscles. The algorithm devised by us for calculation of mass distribution (Kowalczyk \& Skubiszak, 1999) allows, for the first time, direct checking of the correlations between the symmetries of the whole filament and its cross-bridge crowns. The concurrent verification of the two alternative modes might be helpful in elucidation of the existing ambiguities in the interpretation of some experimental data related not only to the filament features but also to the muscle contraction mechanism.

\section{METHODS}

\section{Computation of mass distribution}

Mass distribution within a model is calculated by summarizing all pixels depicting the molecules along the particular lines passing through the 3D model perpendicularly to a plane (Kowalczyk \& Skubiszak, 1999). In this way, a particular picture shows the mass distribution in the chosen direction. The calculation in a direction perpendicular to the filament axis allows comparison of the model mass distribution with the available EM images of muscles. The calculation along the filament axis gives a unique possibility of direct examination of the model symmetry. An essential advantage of the approach used is also the possibility to calculate the Fourier transforms in a very easy and effective way.

Each myosin tail is modelled in two manners: either as a rod of a uniform mass density or as a rod divided into the successively arranged ellipsoids. The former presentation is explored for verification of the models by EM and diffraction patterns of both types - optical and low-angle X-ray. The latter approach 
allows determining the contribution of the $\alpha$-helical coiled-coil structure of myosin tails (Cohen \& Holmes, 1963; Elliott et al., 1968; Chew \& Squire, 1995) into high-angle X-ray diffraction patterns depending on the degree of the tail twisting around the filament axis. Ellipsoids of 0.51-nm and 7.25-nm lengths depict the pitches of the $\alpha$-helices and the coils, respectively. The density of mass within each ellipsoid is assumed to be twice that in the rest of the rod volume.

\section{Fourier transforms}

Fourier transform calculations are based on the principles of Fresnel's diffraction and image processing procedure (Gonzalez \& Wintz, 1987).

$$
F(u, v)=\frac{1}{n} \sum_{x=0}^{n-1} \sum_{y=0}^{n-1} f(x, y) e^{-j 2 \pi(u x+v y) / n}
$$

In Eqn. (1), $f(x, y)$ describes the discrete mass distribution in a model and $F(u, v)$ corresponds to the transform result. The relationships between the arguments are as follows: $\Delta \mathrm{u}=1 / \mathrm{n} \Delta \mathrm{x}$ and $\Delta \mathrm{v}=1 / \mathrm{n} \Delta \mathrm{y}$, where $\mathrm{n}$ is a positive integer. The grid dimensions in the $\mathrm{x}$ - and $\mathrm{y}$-axis, ( $\Delta \mathrm{x}$ and $\Delta \mathrm{y}$, respectively) define the calculation precision.

To use the fast Fourier transform algorithm (Gonzalez \& Wintz, 1987), the mass distribution is calculated in a square with a side equal to a power of 2 . To display the high-frequency terms of the Fourier spectra, the logarithmic transformation $\Delta(u, v)=\log (1+|F(u, v)|)$ (Gonzalez \& Wintz, 1987) is used. Throughout this work, FFT stands for the logarithmic, fast Fourier transform.

For verification by optical diffraction patterns, FFT is calculated on the basis of an image representing mass distribution along one direction. To verify by low-angle X-ray diffraction patterns, the model is rotated around the filament axis by $10^{\circ}$ in the range of $360^{\circ}$, mass distribution and appropriate FFT are calcu- lated along each direction, and then the individual FFTs are averaged.

Calculation of the Fourier transforms by the image processing procedure (Gonzalez \& Wintz, 1987) is so much faster and easier than that based on the equation of Klug et al. (1958) that it might seem to be primitive. However, because the resolution of the FFT spectra depends on precision of modelling, at a proper scale it could give spectra not only bearing better resemblance to those observed experimentally, but also reflecting much more details. An essential advantage of the approach used is that it allows calculations for any model fragment, up to a fragment of the muscle sarcomere in a few minutes. Therefore, different configurations of the cross- bridges depicting successive phases of muscle contraction could be considered. This approach is also more unbiased because it allows defining the model symmetry instead of introducing it a priori. Moreover, by consideration of particular structures incorporation of beam parameters into the calculation becomes unnecessary. Because of that, consideration only the myosin head arrangement justifies comparison of the FFT spectra with optical diffraction patterns, computation of whole myosin molecules with uniform distribution of mass permits verification of the model with low-angle X-ray diffraction patterns, and the model representing only LMM fragments with discrete density of mass can be related to high-angle $\mathrm{X}$-ray diffraction patterns.

The FFT spectra presented in this work were calculated in a square with the side 2048 pixels. That allowed revealing up to the 34 -th layer line in the case of visualization of the myosin molecules with uniform density of mass. For comparison with the experimental patterns, it is enough to show only 9-12 layer lines. For clarity, we present such FFT spectra by relative intensities of the reflections. For the model backbones, the FFT spectra were calculated for the models in which each LMM was computed separately either by $0.51-\mathrm{nm}$ or by $7.25-\mathrm{nm}$ ellipsoids; then the two spectra 
were superimposed. Such approach has allowed revealing all specific reflections observed in the high-angle X-ray diffraction patterns of muscles.

\section{RESULTS AND DISCUSSION}

The new mode for myosin molecule arrangement within the vertebrate skeletal muscle thick filaments, despite being considerably different from that recognized for about the half of a century, has led to the modelling, for the first time, of a complete bipolar structure, the CB model (see: Figs. 3 and 4a in Skubiszak \& Kowalczyk, 2002). In the previous paper we have shown that the appearance of the CB model is similar to that observed in EM images, and the dimensions specific for both the whole filament and myosin subfragments agree well with those measured by different experimental methods (see: Tables 1 and 3 in Skubiszak \& Kowalczyk, 2002). Some features of the CB model contradict, however, the general view, first of all as concerns the relation between the symmetries of the whole filament and its cross-bridge crowns. A stereo pair presentation of the cross-bridge zone (see Fig. 1) shows clearly that the CB model looks two-stranded; the TS model in turn appears three-stranded (not shown).

\section{Cross-bridge crown configuration}

The conclusion concerning the threestranded structure of the vertebrate skeletal muscle thick filaments was chiefly made on the grounds of three kinds of experimental evidence (for a review see: Kensler \& Woodhead, 1995; Squire, 1997; Eakins et al., 2002): (1) the thick filaments reveal a threefold-rotational symmetry; (2) distribution of the head mass can be extrapolated by three helical paths of a $3 \times 43 \mathrm{~nm}$ pitch; and (3) in the optical and low-angle X-ray diffraction patterns prominent meridional reflections are situated on the layer lines corresponding to

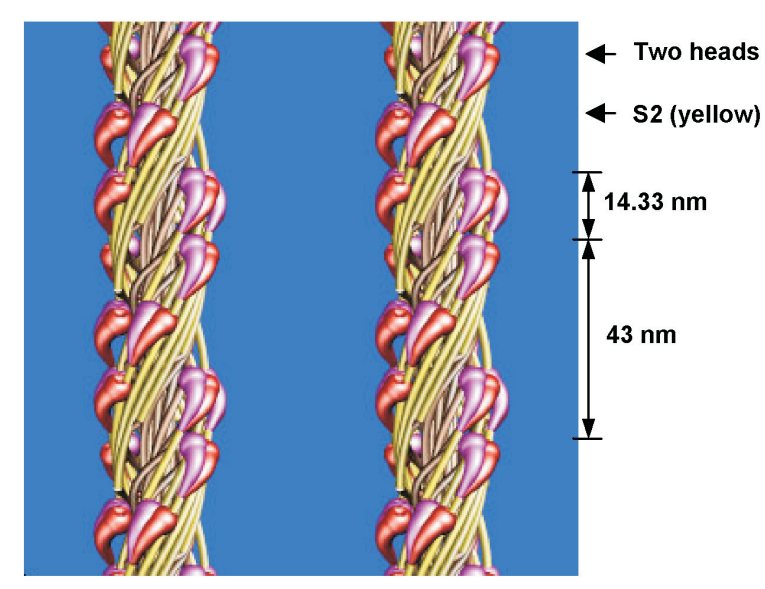

Figure 1. Stereo pair presentation of a crossbridge zone of CB model.

All cross-bridges (each consisting of two myosin heads linked to S2) are arranged on the filament surface along two right-handed helices with a 14.33-nm axial translation and a $2 \times 43-\mathrm{nm}$ pitch. The inclined stripes consisting of the cross-bridges are separated by prominent gaps.

$3 \mathrm{n}$ orders of the $43-\mathrm{nm}$ repeat. Because of that, we have performed the verification of the two models, CB and TS, by analysis of the foregoing features.

The mass distribution calculated along the filament axis allowed direct checking, for the first time, of the model symmetries. This in turn allowed disclosing quite interesting relations. The CB model, despite containing asymmetrical crowns (see: Fig. 5c in Skubiszak \& Kowalczyk, 2002) and looking two-stranded (Fig. 1), reveals true threefold-rotational symmetry (see Fig. 2a). The symmetry is specific for both the filament backbone and surface; this agrees well with the experimental observation (Ashton et al., 1992). In the case of the TS model, each cross-bridge crown has threefold-rotational symmetry (see: Fig. $5 d$ in Skubiszak \& Kowalczyk, 2002), but symmetry of each filament fragment longer than $43 \mathrm{~nm}$ is close to cylindrical (Fig. 2b). Furthermore, it is worth noting that computer simulation of different possible modes of myosin molecule packing has revealed that the symmetries of the filament and its cross-bridge crowns are never the same. 
(a)

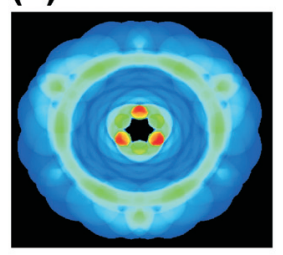

(b)

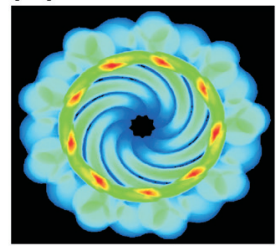

Figure 2. Mass distribution around the filament axis calculated for CB model (a) and TS model (b).

The equilateral triangle in (a) depicts true threefold-rotational symmetry and the circle in (b) indicates cylindrical symmetry. (c) Relative mass density scale.

Despite different symmetries, the mass distribution calculated perpendicularly to the filament axis (see: Fig. 3) can be extrapolated by three symmetrically originating helices of a 3 $\times$ 43-nm pitch for both models, CB and TS, with comparable accuracy. Thus, in this term, both models are consistent with the interpretation of EM data (Kensler \& Stewart, 1983; Kensler \& Woodhead, 1995). However, extrapolation by two helices of a $2 \times 43-n m$ pitch seems to be more reasonable in the case of both the CB model (see Fig. 3a) and the experimental data presented by Cantino and co-workers (see: Fig. 3 in Cantino \& Squire, 1986; or Fig. 2b in Cantino et al., 2002).

The models seem also to be equivalent in terms of the transverse striation. In both models (see Fig. 3), distribution of the myosin head mass reveals grouping into separate transverse stripes (crowns) axially $14.33 \mathrm{~nm}$ apart, and, which is important, the transverse stripes of similar shapes appear every $43 \mathrm{~nm}$. This feature of the models agrees well with that observed for the thick filaments by EM (Kensler \& Stewart, 1983; Cantino \& Squire, 1986; Kensler \& Woodhead, 1995; Cantino et al., 2000; 2002; Eakins et al., 2002) and seems to be crucial for the elucidation of diffraction spectra (see below). This supports also the suggestion (Squire, 1997) that two heads of a myosin molecule are similarly oriented in relation to the myosin tail as distinct from the view (Stewart \& Kensler, 1986; Levine, 1993) that the heads are oriented oppositely.

It is also difficult to give a preference for one of the two models on the ground of a comparison of the FFT calculated by averaging around the filament axis (see Fig. 4) with the low-angle X-ray diffraction patterns of muscles. Concur-

(a)

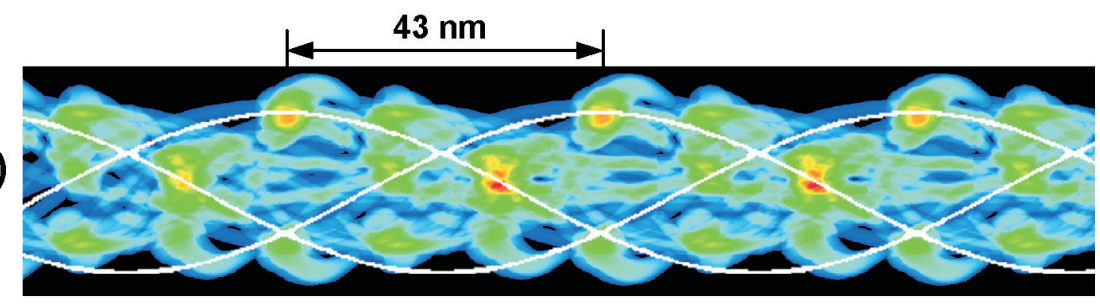

(b)

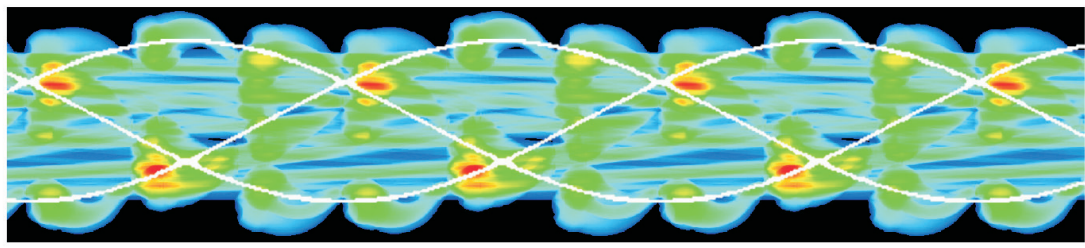

Figure 3. Mass distribution calculated on a plane parallel to the filament axis for CB model (a) and TS model (b).

The scale of relative mass density is the same as in Fig. 2. There is well marked transverse striping axially $14.33 \mathrm{~nm}$ apart. Stripes of the same shape appear $43 \mathrm{~nm}$ apart. The white lines show three symmetrically originating helical paths of a $3 \times 43$-nm pitch by which the mass distribution could be extrapolated. 
(a)

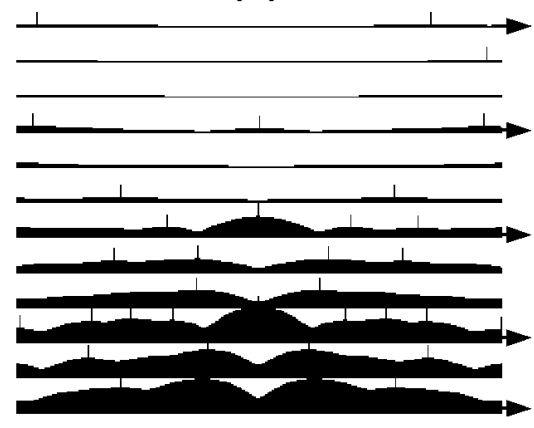

(b)

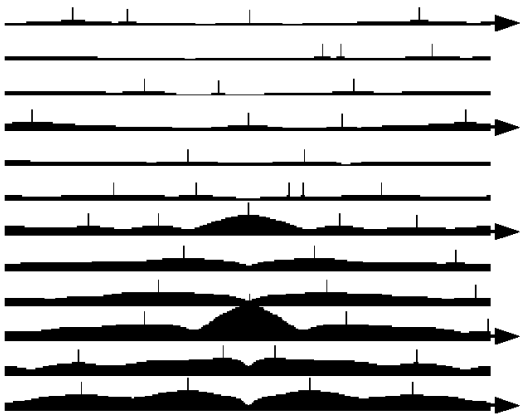

(c)

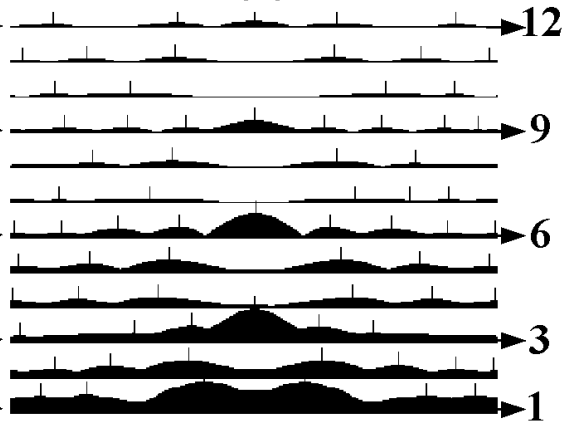

Figure 4. Fourier spectra calculated by averaging around the filament axis: (a) and (b) for the CB model in the case of the whole bipolar structure and a cross-bridge fragment, respectively; (c) for a cross-bridge fragment of the TS model.

The spectra refer to images oriented at $90^{\circ}$ in relation to that shown in Fig. 3.

rently, both models and the thick filaments (compare Fig. 4 with the experimental data in: Huxley \& Brown, 1967; Haselgrove, 1980; Malinchik \& Lednev, 1992; Bordas et al., 1993; $\mathrm{Xu}$ et al., 1997; Linari et al., 2000) give rise to the diffraction patterns in which: (1) all reflections are arranged on the layer lines corresponding to all orders of the 43-nm repeat; (2) the meridional reflections on the layer lines corresponding to $3 \mathrm{n}$ orders of $43 \mathrm{~nm}$, i.e. on the 3rd, 6th, 9th, 12th, etc. ones, are the most prominent, with the stronger reflection on the 3rd layer line corresponding to the 14.33-nm repeat; (3) the reflections on the 1st, 2nd, 4th, and 5th layer lines are less prominent but also quite distinct.

The best detection of the existing distinctions between the CB and TS models was obtained by calculation of the Fourier transforms along one direction perpendicular to the filament axis (see Fig. 5). For the CB model, the FFT spectrum agrees well with the optical diffraction patterns of separate thick filaments (Kensler \& Stewart, 1983; Cantino \& Squire, (a)

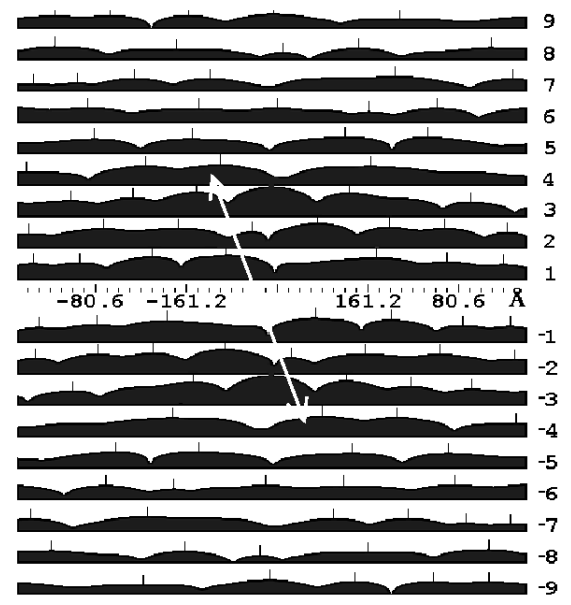

(b)

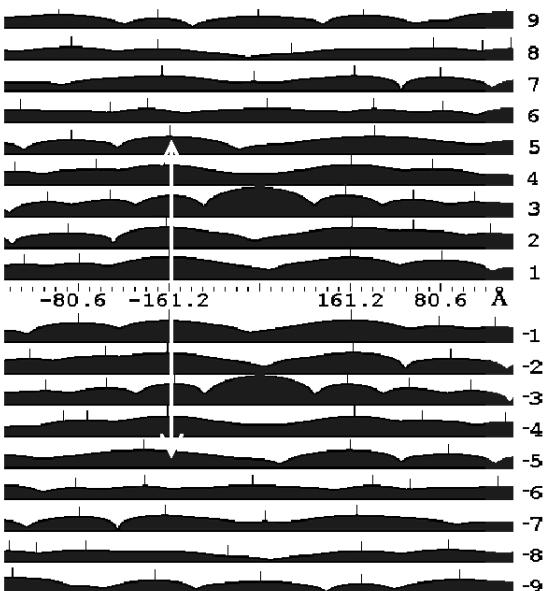

Figure 5. Fourier spectra calculated along one direction perpendicular to the filament axis for a cross-bridge fragment of CB model (a) and TS model (b); the corresponding 3D models are shown in Fig. 4 in Skubiszak \& Kowalczyk (2002).

The spectra refer to images oriented at $90^{\circ}$ in relation to that shown in Fig. 3. The white arrows point on asymmetrical (a) and symmetrical (b) intensity profiles of the FFT spectra. 
1986; Stewart \& Kensler, 1986; Craig et al., 1992; Kensler et al., 1994; Levine, 1997; Cantino et al., 2002) because the intensity profiles are asymmetrical. In the case of the TS model, the profile is nearly symmetrical, i.e. it bears resemblance to the low-angle X-ray diffraction patterns. It is worth noting that the spectrum calculated for the TS model agrees with that calculated for a similar model (compare Fig. 5b with Fig. 1c presented by Eakins et al., 2002), however, the both do not agree with the optical diffraction patterns of the vertebrate skeletal thick filaments. As symmetrical and asymmetrical intensity profiles we mean the arrangement of the reflections on particular layer lines, respectively, one under the other, i.e. symmetrically in relation to the meridian, and in a cross-like manner (see the white arrows in Fig. 5).

The differences, i.e. asymmetrical intensity profile in the case of optical diffraction patterns and symmetrical profile in the case of low-angle X-ray diffraction patterns, are usually elucidated by artifacts possible in the optical method. So, for model verification the low-angle X-ray diffraction patterns are usually considered. However, optical diffraction patterns detect mass distribution on the surface of the cross-bridge fragment of a separate thick filament, and only in the direction of beam propagation. Low-angle X-ray diffraction patterns reflect the mass distribution within a muscle sarcomere volume. Because of that not only the structure of the thick filament, including the filament bipolarity, but also the structure of the accessory proteins, e.g. C-protein, as well as the arrangement of the myofilaments, thick, thin, and titin, within the hexagonal lattice of the muscle sarcomere contribute into the intensity profile of the X-ray spectrum. The intensities of the particular reflections, in turn, depend on the experimental conditions, such as the muscle state, temperature, ionic strength, $\mathrm{pH}$, etc. (Xu et al., 1997). Therefore, it should not be surprising that it is possible to find such disposition of the myosin heads (usually called perturbations, see Methods), which gives rise to a satisfactory consistency of the calculated Fourier transform with the low-angle X-ray diffraction patterns. Similarity of the FFT spectra (see Fig. 4) calculated for the models of different symmetry (CB model looks two-stranded although has true threefold-rotational symmetry; TS model, in turn, looks three-stranded but has a cylindrical symmetry) suggest that comparison of the reflection intensities calculated and observed by low-angle X-ray diffraction should not be considered as decisive for the verification of the thick filament structure, especially if only a polar fragment of the filament model is taken into account. The model depicting a fragment of a separate filament should be verified by the optical diffraction patterns (compare Fig. 5 with optical diffraction patterns in: Kensler \& Stewart, 1983; Cantino \& Squire, 1986; Stewart \& Kensler, 1986; Craig et al., 1992; Kensler et al., 1994; Levine, 1997; Cantino et al., 2002).

The performed analysis also indicates that the main feature of the muscle diffraction patterns, i.e. arrangement of all reflections on separate layer lines $14.33 \mathrm{~nm}$ apart, is determined not by a helical arrangement of the myosin heads on the filament surface (see Fig. 4 in Skubiszak \& Kowalczyk, 2002) but by the transverse striping which is markedly visible when the mass distribution is explored (see Fig. 3). Grouping of the cross-bridge crowns, axially separated by $14.33 \mathrm{~nm}$, in sets of three (see: Fig. 3) should give rise to an increase of the reflections corresponding to the 43-nm repeat, as well as to $3 \mathrm{n}$ order of $43 \mathrm{~nm}$, i.e. the 1st, 3rd, 6th, 9th, 12th, etc. reflections should be prominent with the most prominent one corresponding to the main axial repeat, the 14.33-nm translation between the crowns. Arrangement of the heads along a helix of a pitch $2 \times 43 \mathrm{~nm}$ (see Fig. 1) should bring about an increase of the reflections on the 2 nd and 4 th layer lines. In terms of such interpretation neither of the reflections is forbidden (see Methods). 


\section{Myosin tail configuration}

The mass distributions calculated for the model backbones (see: Fig. 6) have clearly revealed two axial repeats: one corresponding to the 14.33-nm staggers between the successive structure of each myosin tail is simulated (see Methods). The 14.33-nm repeat undoubtedly contributes into the FFT spectrum presented in Figs. 4 and 5 markedly increasing the intensity of reflections on the 3rd layer line.

(a)

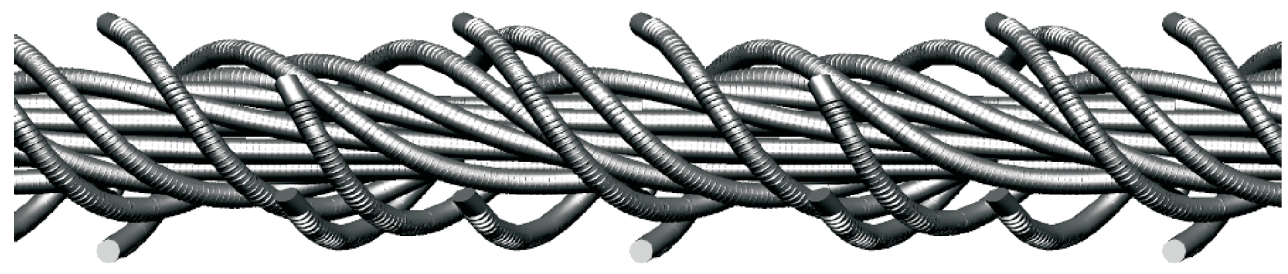

(b)

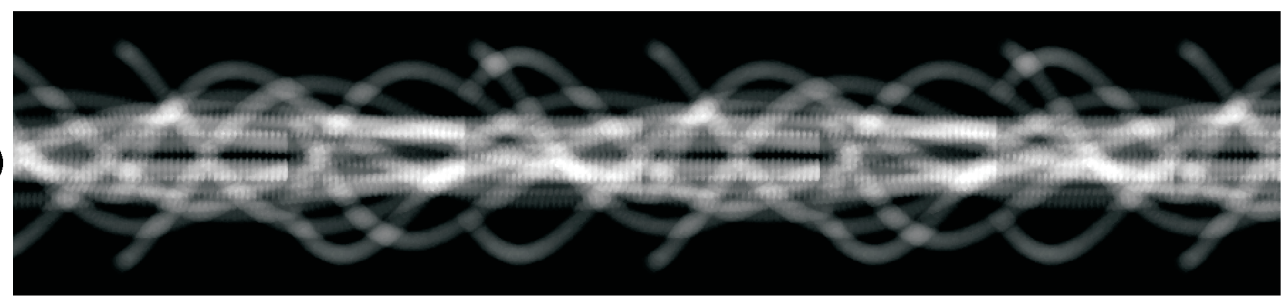

(c)

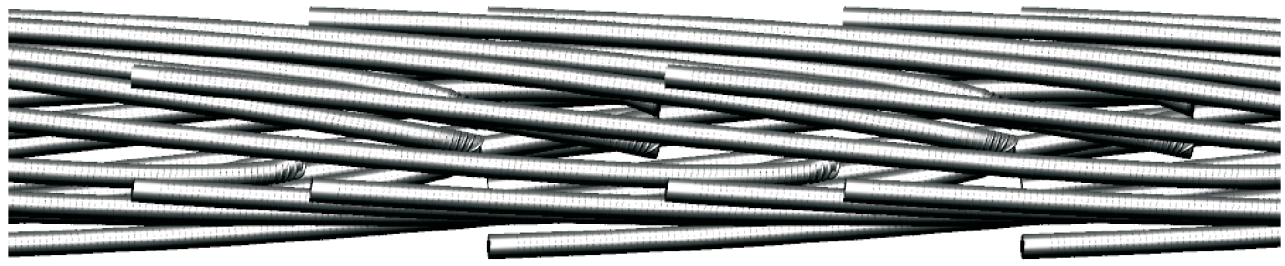

(d)

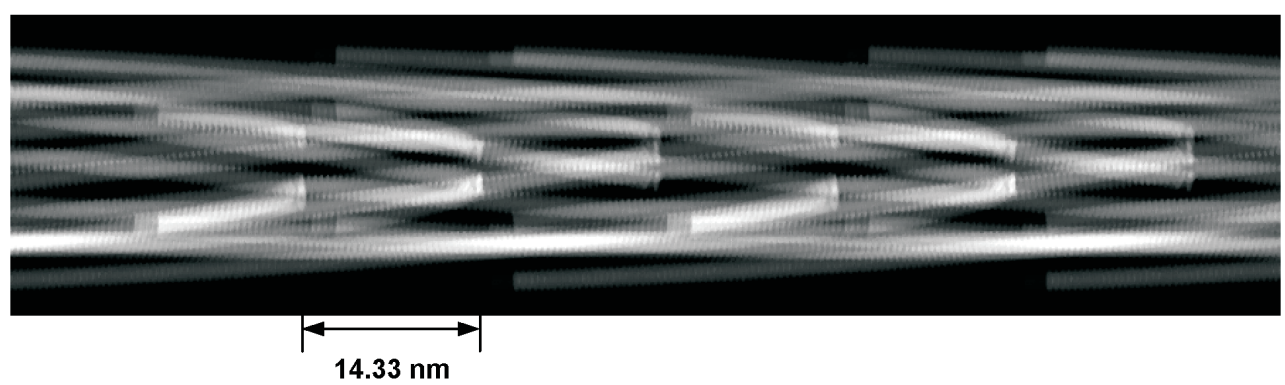

Figure 6. Backbone fragments corresponding to the cross-bridge zones of CB model (a) and TS model (c).

In the case of the TS model, the backbone structure is similar to that modeled by Chew \& Squire (1995) because inclination of the straight tail is about $3^{\circ}$. In both the CB and TS models, the $\alpha$-helical structure of each LMM unit is depicted by successively arranged ellipsoids of 0.51-nm length (see Methods); (b) and (d), mass distributions calculated on a plane parallel to the filament axis for the CB model and the TS model, respectively. The black and white pixels stand for an absence and maximal density of mass, respectively.

tails (see: Fig. 1 in Skubiszak \& Kowalczyk, 2002) and the other - to the axial arrangement of either the 0.51-nm long ellipsoids (see: Figs. 6b and 6d) or the 7.25-nm ones (not shown), by which the $\alpha$-helical coiled-coil
The Fourier spectra calculated for the backbones of the two models (Fig. 7) have a number of similarities. First of all, the spectra reveal the reflections specific for the high-angle X-ray diffraction patterns (Cohen \& Holmes, 
(a)

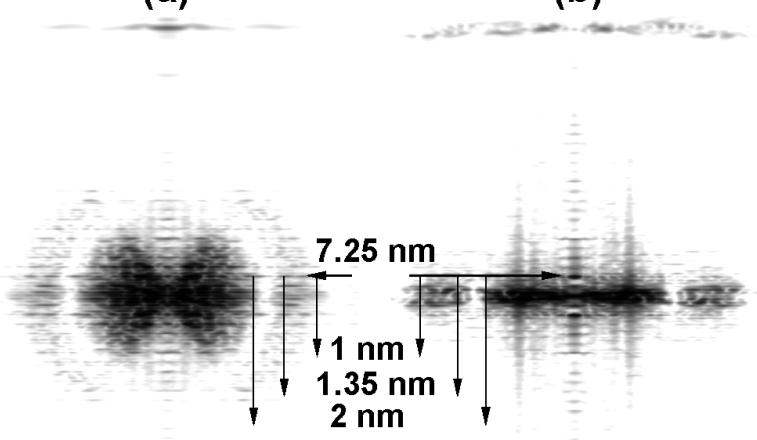

\section{$0.51 \mathrm{~nm}$}

Figure 7. Fourier spectra calculated for the backbone fragments of CB model (a) and TS model (b) oriented at $90^{\circ}$ in relation to that shown in Fig. 6.

The picture represents the result of superimposing of two separate spectra, one reflecting the 0.51-nm units (shown in Fig. 6b and 6d) and the second - the 7.25-nm units (not shown but described in Methods).

1963; Chew \& Squire, 1995), i.e. the meridional reflection at position $1 / 0.51 \mathrm{~nm}^{-1}$ and three equatorial ones at positions of about $1 / 2.0 \mathrm{~nm}^{-1}, 1 / 1.35 \mathrm{~nm}^{-1}$, and $1 / 1.0 \mathrm{~nm}^{-1}$. As was mentioned in the Introduction, the foregoing reflections are usually recognized as a strong argument for the arrangement of the myosin tails parallel, or nearly so, to the filament axis. However, the degree of the tail twisting in the $\mathrm{CB}$ model is considerably stronger than in the TS model (compare Fig. $6 \mathrm{a}$ and $6 \mathrm{c}$ ). In spite of that, the FFT spectrum for the CB model backbone is in better consistency with the experimental data (Chew \& Squire, 1995) than in the case of the TS model because: (1) the $0.51-\mathrm{nm}$ reflection corresponding to the axial repeat in the $\alpha$-helical arrangement of the myosin tail amino acids (see Methods) is precisely on the meridian; in the case of the TS model, it is in an off-meridional position; (2) half of the coiled-coil pitch, 7.25 $\mathrm{nm}$ (see Methods), is reflected by the streak spreading up to the 7.25-nm layer line at the equatorial position $1 / 1.35 \mathrm{~nm}^{-1}$; in the case of the TS model, the streak spreads only to about the 14.5-nm layer line, and the 7.25-nm reflection on the meridian is not observable experimentally. This result suggests that not the whole tail should be arranged near-parallel to the filament axis, with an inclination of about 3 (Chew \& Squire, 1995); it is enough that the C-terminus of about 43-nm length be arranged near-parallel (see: Fig. 6a and Fig. 1 in Skubiszak \& Kowalczyk, 2002), the rest of the tail could be quite strongly twisted.

\section{CONCLUSIONS}

(1) Only twisted myosin tails and asymmetrical cross-bridge crowns could ensure both a tube like appearance and true threefold-rotational symmetry of the whole bipolar filament. (2) The symmetries of the whole filament and each of its cross-bridge crowns cannot be the same. (3) In a longitudinal view, the cross-bridge zone of the filament of true threefold-rotational symmetry looks two-stranded with a pitch of $2 \times 43 \mathrm{~nm}$ although the mass distribution visible in a plane parallel to the filament axis could be quite well extrapolated by three symmetrically originating helical paths with a pitch of $3 \times 43 \mathrm{~nm}$. The crossbridge zone looks three-stranded with a pitch of $3 \times 43 \mathrm{~nm}$ in the case of the filament of cylindrical symmetry. (4) The optical and low-angle X-ray diffraction patterns reflect not a $9 / 3$ helical symmetry of the filament, but the grouping of all myosin heads into crowns the mass distribution of which reveals the sets of three transverse stripes along the filament axis. (5) No perturbation could eliminate the excess in the number of cross-bridges protruding from the filament backbone in the case of the models of the TS type; the symmetrical configuration of the cross-bridge crowns does gives rise to nine cross-bridges visible in a transverse view instead of six observed experimentally (Yu \& Brenner, 1986; Craig et $a l ., 1992)$. (6) To observe the reflections specific for the high-angle X-ray diffraction patterns of the vertebrate skeletal muscles it is 
necessary that both a fragment of about 43 $\mathrm{nm}$ of the C-terminus of each of myosin tails be arranged near-parallel to the filament axis and the rest of the tail be quite strongly twisted.

The CB model, besides better consistency with the experimental data, involves a new manner for the cross-bridge action, by unwrapping along a helical path (Skubiszak, 1993) instead of oar like, by bending of the myosin molecule. In terms of such movement, it becomes comprehensible such phenomena as the generation by muscle of both axial and radial force (Brenner \& Yu, 1991), the elasticity of the muscle sarcomeres without changing of the myofilament lengths (Huxley, 1980), as well as the ideal matching between the specific binding sites on myosin heads and actin monomers required during the continuous relative movement of the thick and thin filaments because of the stereospecificity of the actin-myosin interaction (Holmes, 1997).

\section{R E F E R E N C E S}

Ashton FT, Weisel J, Pepe FA. (1992) The myosin filament. XIV. Backbone structure. Biophys J.; 61: 1513-28.

Bennett PM. (1981) The structure of spindle-shaped paracrystals of light meromyosin. J Mol Biol.; 146: 201-21.

Bordas J, Diakun GP, Diaz FG, Harries JE, Lewis RA, Lowy J, Mant GR, Towns-Andrews E. (1993) Two-dimensional time-resolved X-ray diffraction studies of live isometrically contracting frog sartorius muscle. J Muscle Res Cell Motil.; 14: 311-24.

Brenner B, Yu LC. (1991) Characterization of radial force and radial stiffness in $\mathrm{Ca}^{2+}$-activated skinned fibres of the rabbit psoas muscle. J Physiol.; 441: 703-18.

Cantino M, Squire JM. (1986) Resting myosin cross-bridge configuration in frog muscle thick filaments. J Cell Biol.; 102: 610-18.

Cantino ME, Brown LD, Chew M, Luther PK, Squire JM. (2000) A-band architecture in ver- tebrate skeletal muscle: polarity of the myosin head array. J Muscle Res Cell Motil.; 21: 681-90.

Cantino ME, Chew MW, Luther PK, Morris E, Squire JM. (2002) Structure and nucleotidedependent changes of thick filaments in relaxed and rigor plaice fin muscle. J Struct Biol.; 137: 164-75.

Chew MWK, Squire JM. (1995) Packing of $\alpha$-helical coiled-coil myosin rods in vertebrate muscle thick filaments. J Sruct Biol.; 115: 223-49.

Cohen C, Holmes KC. (1963) X-ray diffraction evidence for $\alpha$-helical coiled-coils in native muscle. J Mol Biol.; 6: 423-32.

Craig R, Alamo L, Padron R. (1992) Structure of the myosin filaments of relaxed and rigor vertebrate striated muscle studied by rapid freezing electron microscopy. J Mol Biol.; 228: 474-87.

Eakins F, AL-Khayat HA, Kensler RW, Morris EP, Squire JM. (2002) 3D structure of fish muscle myosin filaments. J Struct Biol.; 137: 154-63.

Elliott A, Lowy J, Parry DAD, Vibert PJ. (1968) Puzzle of the coiled coils in the $\alpha$-protein paramyosin. Nature.; 218: 656-9.

Gonzalez RC, Wintz P. (1987) Image transforms. In Digital image processing. 61-137. AddisonWesley Publishing Comp. Massachusetts.

Harrison RG, Lowey S, Cohen C. (1971) Assembly of myosin. J Mol Biol.; 59: 531-5.

Haselgrove JCR, Rodger CD. (1980) The interpretation of X-ray diffraction patterns from vertebrate striated muscle. J Musc Res Cell Motil.; 1: 371-90.

Holmes KC. (1997) The swinging lever-arm hypothesis of muscle contraction. Curr Biol.; 7: R112-18.

Hudson L, Harford JJ, Denny RC, Squire JM. (1997) Myosin head configuration in relaxed fish muscle: resting state myosin heads must swing axially by up to $150 \AA$ or turn upside down to reach rigor. $J$ Mol Biol.; 273: 440-55. 
Huxley AF. (1980) Reflection on muscle. Princeton University Press, Princeton, New Jersey.

Huxley HE. (1963) Electron microscope studies on the structure of natural and synthetic protein filaments from striated muscle. $\mathrm{J} \mathrm{Mol}$ Biol.; 7: 281-308.

Huxley HE, Brown W. (1967) The low-angle $\mathrm{X}$-ray diagram of vertebrate striated muscle and its behaviour during contraction and rigor. J Mol Biol.; 30: 383-434.

Ip W, Heuser J. (1983) Direct visualization of the myosin cross-bridge helices on relaxed rabbit psoas thick filaments. J Mol Biol.; 171: $105-9$.

Kensler RW, Stewart M. (1983) Frog skeletal muscle thick filaments are three-stranded. $J$ Cell Biol.; 96: 1797-802.

Kensler RW, Woodhead JL. (1995) The chicken muscle thick filament: temperature and the relaxed cross-bridge arrangement. J Muscle Res Cell Motil.; 16: 79-90.

Kensler RW, Peterson S, Norberg M. (1994) The effects of changes in temperature or ionic strength on isolated rabbit and fish skeletal muscle thick filaments. J Muscle Res Cell Motil.; 15: 69-79.

Klug A, Crick FHC, Wyckoff HW. (1958) Diffraction by helical structures. Acta Cryst.; 11: 199-212.

Kowalczyk L, Skubiszak L. (1999) Algorithm for fast calculation of mass distribution. Biocyb Biomed Eng.; 19: 31-38.

Levine RJC. (1993) Evidence for overlapping myosin heads on relaxed thick filaments of fish, frog, and scallop striated muscles. J Struct Biol.; 110: 99-110.

Levine RJC. (1997) Differences in myosin head arrangement on relaxed thick filaments from Lethocerus and rabbit muscles. J Muscle Res Cell Motil.; 18: 529-43.

Linari M, Piazzesi G, Dobbie I, Koubassova N, Reconditi M, Narayanan T, Diat O, Irving M, Lombardi V. (2000) Interference fine structure and sarcomere length dependence of the axial X-ray pattern from active single muscle fibers. Proc Natl Acad Sci US A.; 97: $7226-31$.

Luther PK, Squire JM. (1980) Three-dimensional structure of the vertebrate muscle A-band. II. The myosin filament superlattice. J Mol Biol.; 141: 409-39.

Malinchik SB, Lednev VJ. (1992) Interpretation of the X-ray diffraction pattern from relaxed skeletal muscle and modelling of the thick filament structure. J Muscle Res Cell Motil.; 13: 406-19.

Malinchik SB, Xu S, Yu LC. (1997) Temperature-induced structural changes in the myosin thick filament of skinned rabbit psoas muscle. Biophys J.; 73: 2304-12.

Podlubnaya ZA, Kalamkarova MB, Nankina VP. (1969) Polymorphism of the light meromyosin crystallization. J Mol Biol.; 46: 591-2.

Rayment I, Rypniewski WR, Schmidt-Bäse K, Smith R, Tomchick DR, Benning MM, Winkelmann DA, Wessenberg G, Holden HM. (1993) Three-dimensional structure of myosin subfragment-1: A molecular motor. Science.; 261: $50-8$.

Skubiszak L. (1993) Mechanism of muscle contraction. Technol Health Care.; 1: 133-42.

Skubiszak L, Kowalczyk L. (2001) Relation between the mechanical properties of muscles and their structure on the molecular level. Eng Trans.; 49: 191-212.

Skubiszak L, Kowalczyk L. (2002) Myosin molecule packing within the vertebrate skeletal muscle thick filaments. A complete bipolar model. Acta Biochim Polon.; 49: 829-40.

Squire JM. (1972) General model of myosin filament structure. 2. Myosin filaments and crossbridge interactions in vertebrate striated and insect flight muscles. J Mol Biol.; 72: $125-38$.

Squire JM. (1973) General models of myosin filament structure. 3. Molecular packing arrangements in myosin filaments. $J$ Mol Biol.; 77: 291-323. 
Squire JM. (1997) Architecture and function in the muscle sarcomere. Curr Opin Struct Biol.; 7: 247-57.

Stewart M, Kensler R. (1986) Arrangement of myosin heads in relaxed thick filaments from frog skeletal muscle. J Mol Biol.; 192: 831-51.

Xu S, Malinchik S, Gilroy D, Kraft T, Brenner B, Yu LC. (1997) X-ray diffraction studies of cross-bridges weakly bound to actin in relaxed skinned fibers of rabbit psoas muscle. Biophys J.; 73: 2292-303.
Yagi N, Offer GW. (1981) X-ray diffraction and electron microscopy of a light meromyosin tactoid. J Mol Biol.; 151: 467-90.

Yagi N, O’Brien EJ, Matsubara I. (1981) Changes of thick filament structure during contraction of frog striated muscle. Biophys J.; 33: 121-38.

Yu LC, Brenner B. (1986) High-resolution equatorial X-ray diffraction from single skinned rabbit psoas fibers. Biophys J.; 49: 133-5. 